\title{
Postoperative pain, nausea and vomiting among pre- and postmenopausal women undergoing cystocele and rectocele repair surgery
}

\author{
Sepideh Vahabi, Abolfazl Abaszadeh, Fatemeh Yari, and Nazanin Yousefi \\ Department of Anesthesiology and Critical Care, Lorestan University of Medical Science, Khoramabad, Iran
}

\begin{abstract}
Background: Postoperative nausea and vomiting (PONV) and postoperative pain are among the most common sideeffects of surgery. Many factors, such as a change in the level of sex hormones, are reported to affect these complications. This study aimed to evaluate the probable effects of the menopause on PONV and postoperative pain.

Methods: Prospective study, in which a total number of 144 female patients undergoing cystocele or rectocele repair surgery under standardized spinal anesthesia were included. Patients were divided into two equally sized sample groups of pre- and postmenopausal women $(\mathrm{n}=72)$. The occurrence of PONV, the severity of pain as assessed by visual analog scale (VAS) pain score, and the quantity of morphine and metoclopramide required were recorded at 2, 4, 6, 12, 18 and $24 \mathrm{~h}$ after surgery.

Results: The mean VAS pain score and the mean quantity of morphine required was higher among premenopausal women $(\mathrm{P}=0.006)$. Moreover, these patients required more morphine for their pain management during the first $24 \mathrm{~h}$ after surgery compared to postmenopausal women $(\mathrm{P}<0.0001)$. No difference was observed between the two groups regarding the incidence of $\mathrm{PONV}(\mathrm{P}=0.09$ and $\mathrm{P}=1.00$ for nausea and vomiting, respectively) and the mean amount of metoclopramide required $(\mathrm{P}=0.38)$.

Conclusions: Premenopausal women are more likely to suffer from postoperative pain after cystocele and rectocele repair surgery. Further studies regarding the measurement of hormonal changes among surgical patients in both pre- and postmenopausal women are recommended to evaluate the effects on PONV and postoperative pain.
\end{abstract}

Key Words: Menopause, Postoperative nausea and vomiting, Postoperative pain.

Corresponding author: Sepideh Vahabi, M.D.

Department of Anesthesiology and Critical Care, Lorestan University of Medical Science, Kamalvand St, Khoramabad 11111, Iran

Tel: 98-9375347941, Fax: 98-9375347941

E-mail: swt_1392@farasa.org

Received: August 14, 2014.

Revised: 1st, November 4, 2014; 2nd, December 21, 2014.

Accepted: December 31, 2014.

Korean J Anesthesiol 2015 December 68(6): 581-585

http://dx.doi.org/10.4097/kjae.2015.68.6.581

\section{Introduction}

Postoperative nausea and vomiting (PONV) and postoperative pain are common side-effects of surgery $[1,2]$. PONV is reported in $20-30 \%$ of patients undergoing surgery, resulting in a longer hospital stay, dehydration, electrolyte imbalance and suture line bleeding after the surgery causing patient discomfort, morbidity and increased medical costs [3]. Multiple factors are reported to affect the incidence of PONV, including the method of anesthesia, the type of the surgery, patient characteristics such

(c) This is an open-access article distributed under the terms of the Creative Commons Attribution Non-Commercial License (http://creativecommons.org/ licenses/by-nc/4.0/), which permits unrestricted non-commercial use, distribution, and reproduction in any medium, provided the original work is properly cited. 
as sex, age, body weight and stage of the menstrual cycle [4]. A higher incidence of PONV is reported among females [4]. This increase in the frequency of PONV, as well as its variation during the menstrual cycle is considered to be affected by fluctuations of the sex hormones in females. Moreover, several studies have suggested that younger patients and those with higher rates of body fat are at increased risk of PONV [4].

Postoperative pain is a predictable and manageable physiologic response to tissue injury occurring in up to $75 \%$ of surgical patients [5]. Increased awareness regarding the prevalence of postoperative pain and recent advances in the field of pain physiology have resulted in a more sophisticated approach toward pain management during surgical procedures [5]. Ineffective management of postoperative pain is known to be associated with, most notably, patient dissatisfaction and delayed hospital discharge [2,5].

Although most of the studies evaluating the association between the menstrual cycle and PONV consider high levels of the sex hormones, most notably, estrogen as a predisposing factor for PONV [3], some authors disagree since the incidence of PONV is higher among females even after the menopause [6]. Moreover, the results of multiple studies performed to assess the association between postoperative pain and the menstrual cycle are not in agreement $[2,4]$. While the menopause is reported to reduce the severity of pain in some patients, it showed a worsening effect in others [7].

The current study was performed to evaluate the incidence of PONV and the severity of postoperative pain among pre- and postmenopausal women during the first $24 \mathrm{~h}$ after cystocele or rectocele repair surgery. The probable effects of age and body mass index (BMI) on PONV and postoperative pain were also assessed.

\section{Materials and Methods}

\section{Patient enrollment}

The current prospective study was performed at the Asali Hospital, Khoram abad- Lorestan, Iran between March 2012 and March 2013. Female patients undergoing cystocele and rectocele repair surgery aged over 30 years were included. Exclusion criteria were as follows: a) taking hormone therapy or oral contraceptive pills, b) chronic diseases including diabetes mellitus, high blood pressure, hepatic or renal failure, cancer, c) a history of drug addiction, d) amenorrhea and menstrual cycle irregularity in a premenopausal woman, e) pregnancy or breastfeeding in a premenopausal women, $\mathrm{f}$ ) inability to communicate to evaluate pain severity or the occurrence of nausea and vomiting, and g) preoperative American Society of Anesthesiologists score III or higher [8]. A minimum sample size of 60 patients in each group was calculated with a confidence level of $90 \%$, and power of $80 \%$ using "formula for sample size calculation for comparison between two groups when endpoint is quantitative data". The mean visual analog scale (VAS) pain score was considered the desirable outcome. Predicting drop-out rates of $20 \%, 12$ cases were added to the sample size of each group making the number of cases in each group 72. Therefore, a total of 144 female patients were enrolled in this study using consecutive sampling. Patients were divided into two groups of pre-and postmenopausal women $(\mathrm{n}=72)$ according to their menopausal status. The current study was approved by the Research Council and the Ethics Committee of Lorestan University of Medical Science. The investigations were explained to all patients before they were entered into the study and were asked to provide informed consent. None of the patients withdrew from the study during this process.

\section{Measurements}

Patients' characteristics including age, BMI and a detailed medical history were recorded before surgery. After entering the operating room, a peripheral venous access was established using an 18-gauge catheter. Ringer lactate solution was administrated with a volume of $10 \mathrm{ml} / \mathrm{kg}$. Spinal anesthesia was induced by an anesthesiologist according to the standard protocol, injecting 5\% lidocaine with a dosage of 25-50 mg through a 25-gauge catheter placed in the intraspinal space between lumbar vertebrae 3 and 4 (L3 and L4) or 4 and 5 (L4 and L5).

The severity of pain was assessed using the VAS with a minimum score of 0 and maximum score of 10 . Pain severity and the occurrence of nausea and vomiting were assessed at 2, 4, 6, 12,18 and $24 \mathrm{~h}$ after surgery by a blinded observed. If a patient's VAS pain score was higher than 5 , morphine would be administrated at a dose of $0.1 \mathrm{mg} / \mathrm{kg}$ via intramuscular injection. The VAS pain score, the occurrence of nausea and vomiting, and the amount of administrated drugs during the first $24 \mathrm{~h}$ period of follow-up were documented.

\section{Data analysis}

Data were analyzed using the SPSS software package version 20 (IBM, New York, USA). Data are presented as means \pm standard deviation. Repeated-measures ANOVA was used to compare the pain severity between the two groups. The incidence of nausea and vomiting was compared between the two groups using the chi-squared test or Fisher's exact test as indicated. The independent t-test was performed to compare the amount of drugs administered after the surgery between the two groups. A P value lower than 0.05 was considered to indicate statistical significance. 


\section{Results}

\section{Patients' characteristics}

The mean age of the enrolled patients was significantly lower in premenopausal compared to postmenopausal women (43.3 \pm 4.5 vs. $58.9 \pm 5.1$ years, and $\mathrm{P}<0.05$ ) while there was no significant difference between the two groups regarding the BMI $(26.3 \pm 4.2$ vs. $26.1 \pm 4.1$, and $\mathrm{P}=0.81)$. Table 1 summarizes the characteristics of the patients in both groups.

\section{PONV}

The frequency of PONV was not significantly different between the two groups ( $\mathrm{P}=0.86$ and 1.0 , respectively). Postoperative nausea was observed in $40.3 \%$ of premenopausal women and $41.7 \%$ of postmenopausal women. Postoperative vomiting was observed in $36.1 \%$ of women in both groups. Patients in both groups were divided into four subgroups according to their BMI $(<18,18-24.9,25-29.9$, and $>30)$. No difference in the frequency of PONV was observed among patients in the different BMI groups in pre- and postmenopausal women (Fisher's exact test). The participants in each group of pre- and postmenopausal women were also divided into two subgroups according to their age: 30-39 years and 40-49 years for premenopausal women, and 50-59 years and 60-69 years for postmenopausal women. No difference in the frequency of PONV was observed between these subgroups of pre- and postmenopausal women (chi-squared test).

\section{Postoperative pain}

Repeated-measures ANOVA was performed to compare the mean VAS pain score between pre- and postmenopausal women at the indicated time points. There was a statistically significant difference between the two groups at all time points $(\mathrm{P}=0.006)$ and between the various time points in both groups $(\mathrm{P}<0.0001)$. Fig. 1 shows the mean VAS pain scores in pre- and postmeno- pausal women at 2, 4, 6, 12, 18 and $24 \mathrm{~h}$ after surgery. While there was a significant difference in the mean VAS pain score between various time points in all BMI subgroups $(\mathrm{P}<0.0001)$, there was no difference in the mean VAS pain score between these BMI subgroups among pre- and postmenopausal women $(\mathrm{P}=0.13)$.

Repeated-measures ANOVA revealed that while there was a significant difference in the mean VAS pain score between various time points in all age subgroups $(\mathrm{P}<0.0001)$, there was no difference in the mean VAS pain score between these subgroups among pre- and postmenopausal women $(\mathrm{P}=0.7)$.

\section{Morphine and metoclopramide requirements}

The mean dose of morphine administered during the first $24 \mathrm{~h}$ after surgery was significantly higher in premenopausal compared to postmenopausal women $(8.1 \pm 1.16$ vs. $2.8 \pm 0.5$ $\mathrm{mg}$, and $\mathrm{P}=0.001$ ). Moreover, a significantly greater number of premenopausal women needed morphine injection compared to postmenopausal women ( 80.6 vs. $50 \%$, and $\mathrm{P}<0.0001)$. No significant difference was observed between the two groups re-

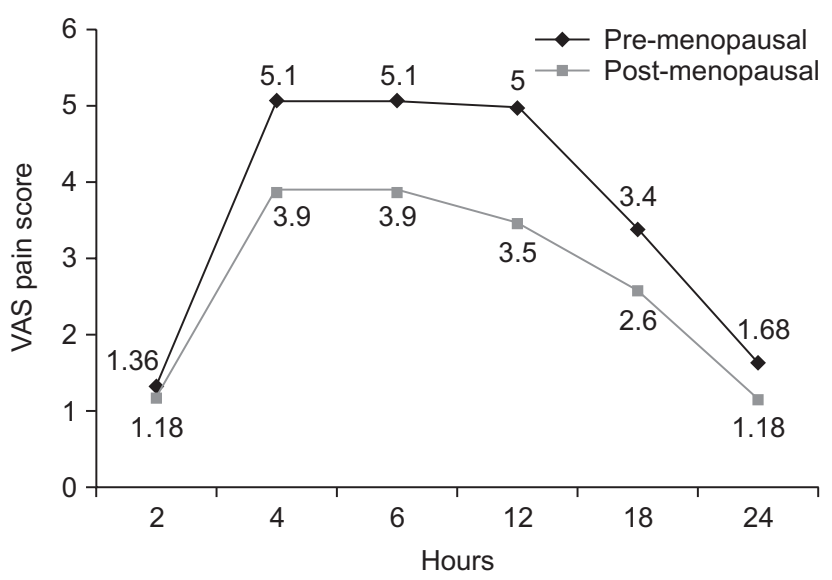

Fig. 1. Mean VAS pain score at 2, 4, 6, 12, 18 and $24 \mathrm{~h}$ after the surgery $(\mathrm{P}=0.006)$.

Table 1. Comparison of Age, BMI, PONV, and Administered Medications between Pre- and Postmenopausal Women

\begin{tabular}{|c|c|c|c|}
\hline & Premenopausal & Postmenopausal & $P$ value \\
\hline Age (yr) & $43.3 \pm 4.5$ & $58.9 \pm 5.1$ & $<0.05^{*}$ \\
\hline $\mathrm{BMI}$ & $26.3 \pm 4.2$ & $26.1 \pm 4.1$ & 0.81 \\
\hline Postoperative nausea (\%) & 40.3 & 41.7 & 0.86 \\
\hline Postoperative vomiting (\%) & 36.1 & 36.1 & 1 \\
\hline Morphine intake in first $24 \mathrm{~h}$ after surgery (mg) & $8.1 \pm 1.16$ & $2.8 \pm 0.5$ & $0.001 *$ \\
\hline Patients needing morphine $(\%)$ & 80.6 & 50 & $<0.0001^{*}$ \\
\hline Metoclopramide intake in first $24 \mathrm{~h}$ after surgery (mg) & $4.4 \pm 0.5$ & $3.4 \pm 0.57$ & 0.28 \\
\hline Patients needing metoclopramide (\%) & 40.3 & 33.3 & 0.38 \\
\hline
\end{tabular}

Data are presented as means \pm SD or percentage. BMI: body mass index, PONV: postoperative nausea and vomiting. $* \mathrm{P}<0.05$. 
garding the mean dose of metoclopramide administered or the number of patients who needed metoclopramide injection.

\section{Discussion}

According to the results of the current study, the severity of postoperative pain after cystocele and rectocele repair surgery was higher among premenopausal women. Moreover, these patients required a higher analgesic intake for their pain management compared to postmenopausal women. This phenomenon is probably due to the changes in the amount of sex hormones, most importantly, estrogen. It is suggested by some authors that estrogen has a role in the neuronal repair process by modulating nerve growth factor [9]. Moreover, estrogen is known to play an important role in the modulation of pain sensation in the vaginal area [4]. However, the results of several studies indicate that estrogen interacts with drug metabolism [10]. In a study performed by Ji et al. [11], the efficacy of morphine in the management of visceral pain was lower in female rats compared to males. They suggested that estrogen reduces the peripheral effects of morphine [11]. This may explain the results of our study in which postmenopausal women needed lower amounts of morphine after undergoing either rectocele or cystocele repair surgery, probably due to the lower estrogen levels in these patients.

In contrast, other studies have revealed an increased sense of skeletal pain in postmenopausal women due to the lack of estrogen in these individuals, which responded well to hormone replacement therapy [12]. Moreover, in a study performed by Blacklock et al. [13] regarding the modulatory role of estrogen on gene expression of neuronal peptides in dorsal root ganglions, estrogen replacement therapy of ovariectomized female rats resulted in a decreased severity of pain.

It has been stated previously that the diverse effects of estrogen on pain may be due to the variety in the nature of these pains [7]. In a study by Meriggiola et al. [7] to evaluate the probable differences in the quantity and quality of pain among preand postmenopausal Italian women, menopause transition was associated with improvement in headache, osteoarticular pain, and chronic pains of the cervical or the lumbar spine, while it was associated with worsening of other types of moderate pain. These findings indicate that fluctuations in the amount of sex hormones, especially estrogen, have various effects on the severity of pain according to the site and nature of the pain [7].

The results of the current study show no difference between pre- and postmenopausal women regarding the occurrence of PONV and the intake of metoclopramide required for its management. In contrast to our findings, Leksowski et al. [14] reported a higher prevalence of PONV among postmenopausal women. Estrogen is generally thought to be associated with PONV due to the fact that its oral administration may cause vomiting [15]. Moreover, association between vomiting during pregnancy and the high level of sex hormones is well described in the literature [16] indicating that changes in these hormones, especially estrogen, play a role in vomiting in various conditions [16]. However, it has been suggested by Becker [6] that since the higher risk of PONV among females compared to males persists even after the menopause, estrogen does not play a fundamental role in PONV.

In a study performed by Simurina et al. [17] to evaluate the probable effect of the menstrual cycle on the incidence of PONV among Croatian female surgical patients, women in the luteal phase of the menstrual cycle were less likely to suffer from PONV compared to the rest of the study population. In contrast, in another study by Sener et al. [4], performed to investigate the association between various phases of the menstrual cycle with postoperative pain and PONV among Turkish female surgical patients, the frequency of PONV, as well as postoperative pain was highest among women in the luteal and the follicular phases of the menstrual cycle. These patients also required more analgesics and antiemetic medication [4]. The findings of our study are in contrast with both previous reports since no association between PONV and the intake of metoclopramide with the menopausal condition was observed. This difference is most probably due to the fact that unlike our study, general anesthesia was used and the population included only premenopausal women.

Although the current study provides data regarding the differences in PONV and postoperative pain among pre- and postmenopausal women, as well as differences in the severity of pain at various time points after surgery, several limitations should be noted. The levels of sex hormones were not measured during this study and thus no direct conclusion can be reached regarding the effects of these hormones on PONV and postoperative pain. Moreover, it has been reported that the pain threshold may be influenced by age [18]. Due to the small number of pre- and postmenopausal women in the same age group, we were unable to investigate the effect of age on our findings.

According to the results of this study, premenopausal women are more likely to suffer from postoperative pain. Further studies regarding the hormonal changes among female surgical patients in both pre- and postmenopausal women with adjustments for possible influential factors such as age are recommended to evaluate the effect on PONV and postoperative pain. 


\section{References}

1. Eberhart LH, Frank S, Lange H, Morin AM, Scherag A, Wulf H, et al. Systematic review on the recurrence of postoperative nausea and vomiting after a first episode in the recovery room - implications for the treatment of PONV and related clinical trials. BMC Anesthesiol 2006; 6: 14 .

2. Couceiro TC, Valença MM, Lima LC, de Menezes TC, Raposo MC. Prevalence and influence of gender, age, and type of surgery on postoperative pain. Rev Bras Anestesiol 2009; 59: 314-20.

3. Apfel CC, Heidrich FM, Jukar-Rao S, Jalota L, Hornuss C, Whelan RP, et al. Evidence-based analysis of risk factors for postoperative nausea and vomiting. Br J Anaesth 2012; 109: 742-53.

4. Sener EB, Kocamanoglu S, Cetinkaya MB, Ustun E, Bildik E, Tur A. Effects of menstrual cycle on postoperative analgesic requirements, agitation, incidence of nausea and vomiting after gynecological laparoscopy. Gynecol Obstet Invest 2005; 59: 49-53.

5. Apfelbaum JL, Chen C, Mehta SS, Gan TJ. Postoperative pain experience: results from a national survey suggest postoperative pain continues to be undermanaged. Anesth Analg 2003; 97: 534-40.

6. Becker DE. Nausea, vomiting, and hiccups: a review of mechanisms and treatment. Anesth Prog 2010; 57: 150-6.

7. Meriggiola MC, Nanni M, Bachiocco V, Vodo S, Aloisi AM. Menopause affects pain depending on pain type and characteristics. Menopause 2012; 19: 517-23.

8. Rozner MA. The American Society of Anesthesiologists physical status score and risk of perioperative infection. JAMA 1996; 275 : 1544.

9. Smith YR, Zubieta JK. Neuroimaging of aging and estrogen effects on central nervous system physiology. Fertil Steril 2001; 76: 651-9.

10. Cepeda MS, Carr DB. Women experience more pain and require more morphine than men to achieve a similar degree of analgesia. Anesth Analg 2003; 97: 1464-8.

11. Ji Y, Murphy AZ, Traub RJ. Estrogen modulation of morphine analgesia of visceral pain in female rats is supraspinally and peripherally mediated. J Pain 2007; 8: 494-502.

12. Yu SB, Wang MQ, Li YQ, Lv X, Jiang Y, Dong GY, et al. The effects of age and sex on the expression of oestrogen and its receptors in rat mandibular condylar cartilages. Arch Oral Biol 2009; 54: 479-85.

13. Blacklock AD, Johnson MS, Krizsan-Agbas D, Smith PG. Estrogen increases sensory nociceptor neuritogenesis in vitro by a direct, nerve growth factor-independent mechanism. Eur J Neurosci 2005; 21: 2320-8.

14. Leksowski K, Peryga P, Szyca R. Ondansetron, metoclopramid, dexamethason, and their combinations compared for the prevention of postoperative nausea and vomiting in patients undergoing laparoscopic cholecystectomy: a prospective randomized study. Surg Endosc 2006; 20: 878-82.

15. Edlow AG, Bartz D. Hormonal contraceptive options for women with headache: a review of the evidence. Rev Obstet Gynecol 2010; 3: 5565.

16. Lagiou P, Tamimi R, Mucci LA, Trichopoulos D, Adami HO, Hsieh CC. Nausea and vomiting in pregnancy in relation to prolactin, estrogens, and progesterone: a prospective study. Obstet Gynecol 2003; 101: 639-44.

17. Simurina T, Mraovic B, Skitarelic N, Andabaka T, Sonicki Z. Influence of the menstrual cycle on the incidence of nausea and vomiting after laparoscopic gynecological surgery: a pilot study. J Clin Anesth 2012; 24: 185-92.

18. Guirro RR, Guirro EC, de Sousa NT. Sensory and motor thresholds of transcutaneous electrical stimulation are influenced by gender and age. PM R 2015; 7: 42-7. 